\title{
LOW ENERGY TRANSFER TO THE MOON
}

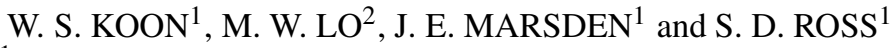 \\ ${ }^{1}$ Control and Dynamical Systems, Caltech, Pasadena, California, U.S.A. \\ ${ }^{2}$ Navigation and Mission Design, Jet Propulsion Laboratory, Pasadena, California, U.S.A.
}

\begin{abstract}
In 1991, the Japanese Hiten mission used a low energy transfer with a ballistic capture at the Moon which required less $\Delta V$ than a standard Hohmann transfer. In this paper, we apply the dynamical systems techniques developed in our earlier work to reproduce systematically a Hiten-like mission. We approximate the Sun-Earth-Moon-spacecraft 4-body system as two 3-body systems. Using the invariant manifold structures of the Lagrange points of the 3-body systems, we are able to construct low energy transfer trajectories from the Earth which execute ballistic capture at the Moon. The techniques used in the design and construction of this trajectory may be applied in many situations.
\end{abstract}

Key words: earth-moon transfer, invariant manifolds, three-body problem, space mission design

\section{How to Get to the Moon Cheaply}

\subsection{HITEN MISSION}

The traditional approach to construct a spacecraft transfer trajectory to the moon from the Earth is by Hohmann transfer. This type of transfer uses only 2-body dynamics. It is constructed by determining a two-body Keplerian ellipse from an Earth parking orbit to the orbit of the moon. See Figure 1(a). The two bodies involved are the Earth and a spacecraft. Such a transfer requires a large $\Delta V$ for the spacecraft to catch up and get captured by the moon.

In 1991, the Japanese mission, Muses-A, whose propellant budget did not permit it to transfer to the moon via the usual method was given a new life with an innovative trajectory design, based on the work of Belbruno and Miller (1993). Its re-incarnation, renamed Hiten, used a low energy transfer with a ballistic capture at the moon. An Earth-to-Moon trajectory of this type, which utilizes the perturbation by the Sun, requires less fuel than the usual Hohmann transfer. See Figures 1(b) and (c).

\subsection{COUPLED THREE-BODY MODEL}

In this paper, we present an approach to the problem of the orbital dynamics of this interesting trajectory by implementing in a systematic way the view that the Sun-Earth-Moon-spacecraft 4-body system can be approximated as two coupled 3-body systems. Below is a schematic of this trajectory in the Sun-Earth rotating 


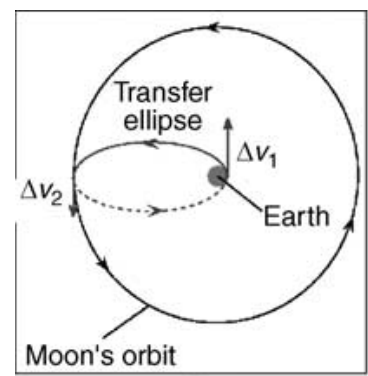

(a)

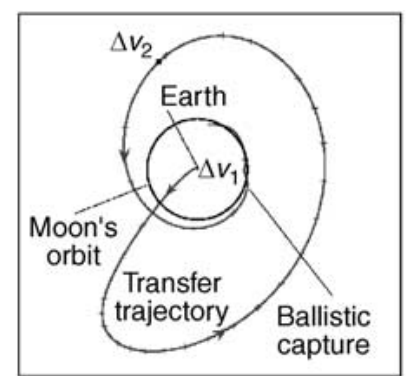

(b)

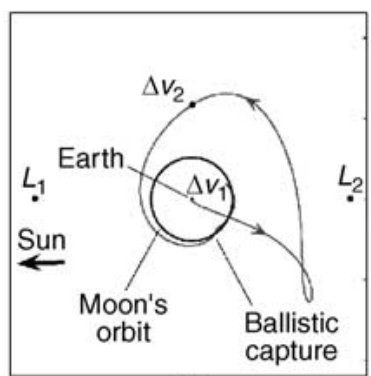

(c)

Figure 1. (a) Hohmann transfer. (b) Low energy transfer trajectory in the geocentric inertial frame. (c) Same trajectory in the Sun-Earth rotating frame.

frame, showing the two legs of the trajectory: (1) the Sun-Earth Lagrange point portion and (2) the lunar capture portion. See Figure 2(a).

Within each 3-body system, using our understanding of the invariant manifold structures associated with the Lagrange points $L_{1}$ and $L_{2}$, we transfer from a $200 \mathrm{~km}$ altitude Earth orbit into the region where the invariant manifold structure of the Sun-Earth Lagrange points interact with the invariant manifold structure of the Earth-Moon Lagrange points. See Figure 2(b). We utilize the sensitivity of the 'twisting' of trajectories near the invariant manifold tubes in the Lagrange point region to find a fuel efficient transfer from the Sun-Earth system to the EarthMoon system. The invariant manifold tubes of the Earth-Moon system provide the dynamical channels in phase space that enable ballistic captures of the spacecraft by the Moon.

The final Earth-to-Moon trajectory is integrated in the bi-circular 4-body model where both the Moon and the Earth are assumed to move in circular orbits about the Earth and the Sun respectively in the ecliptic, and the spacecraft is an infinitesimal mass point. This bi-circular solution has been differentially corrected to

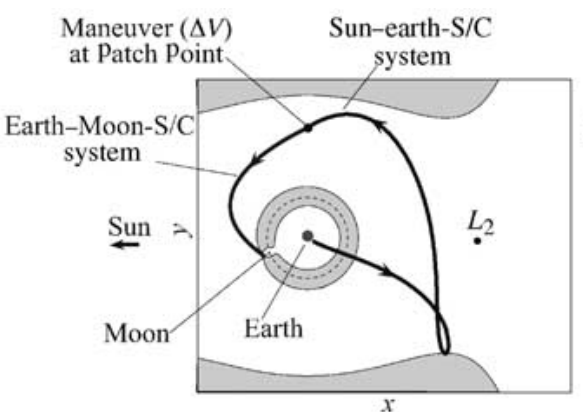

(a)

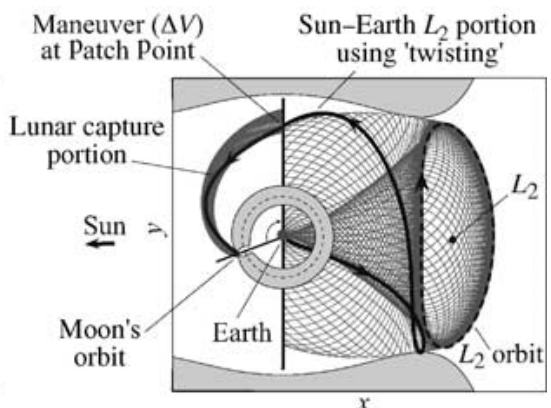

(b)

Figure 2. (a) Two legs of a Hiten-like trajectory in the Sun-Earth rotating frame. (b) The interaction of invariant manifold tubes of the Sun-Earth and the Earth-Moon systems permits a fuel efficient Earth-to-Moon transfer with the perturbation of the Sun. 
a fully integrated trajectory with the JPL ephemeris using JPL's LTool (libration point mission design tool). LTool is JPL's new mission design tool currently under development which is based on dynamical systems theory. This will be described in a subsequent paper.

\section{Planar Circular Restricted Three-Body Problem-PCR3BP}

We start with the PCR3BP as our first model of the mission design space. Let us recall some basic features of this model summarized in Koon et al. (2000).

\subsection{THREE-BODY MODEL}

The PCR3BP describes the motion of a body moving in the gravitational field of two main bodies (the primaries) that are moving in circles. The two main bodies could be the Sun and Earth, or the Earth and Moon, etc. The total mass is normalized to 1 ; they are denoted $m_{\mathrm{S}}=1-\mu$ and $m_{\mathrm{E}}=\mu$. The two main bodies rotate in the plane in circles counterclockwise about their common center of mass and with angular velocity also normalized to 1 . In the actual solar system: the Moon's eccentricity is 0.055 and Earth's is 0.017 which are quite close to circular. The third body, the spacecraft, has an infinitesimal mass and is free to move in the plane. The planar restricted 3-body problem is used for simplicity. Generalization to the 3-dimensional problem is of course important, but many of the essential dynamics can be captured well with the planar model.

\subsection{EQUATIONS OF MOTION}

Choose a rotating coordinate system so that the origin is at the center of mass, the Sun and Earth are on the $x$-axis at the points $(-\mu, 0)$ and $(1-\mu, 0)$ respectively, that is, the distance from the Sun to Earth is normalized to be 1. See Figure 3(a). Let $(x, y)$ be the position of the spacecraft in the plane, then the equations of motion are

$$
\ddot{x}-2 \dot{y}=\Omega_{x}, \quad \ddot{y}+2 \dot{x}=\Omega_{y},
$$

where $\Omega=\left(x^{2}+y^{2}\right) / 2+(1-\mu) / r_{\mathrm{s}}+\mu / r_{\mathrm{e}}$. Here, the subscripts of $\Omega$ denote partial differentiation in the variable. $r_{\mathrm{s}}, r_{\mathrm{e}}$ are the distances from the spacecraft to the Sun and the Earth respectively. See Szebehely (1967) for the derivation.

\subsection{ENERGY MANIFOLDS}

The system of equations is autonomous and can be put into Hamiltonian form with 2 degrees of freedom. It has an energy integral

$$
E=\frac{1}{2}\left(\dot{x}^{2}+\dot{y}^{2}\right)-\Omega(x, y),
$$




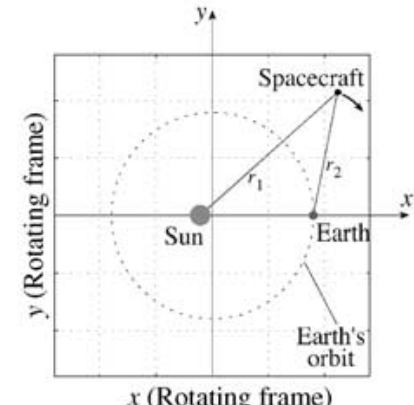

(a)

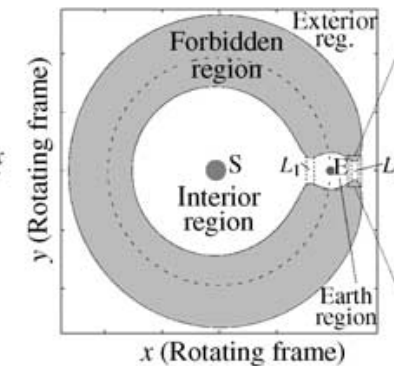

(b)

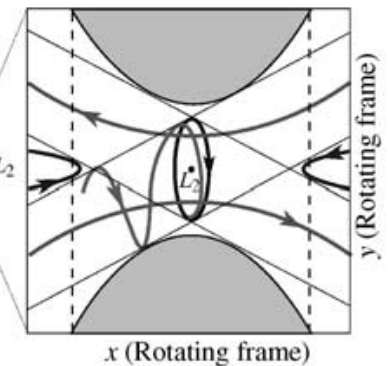

(c)

Figure 3. (a) Equilibrium points of the PCR3BP in the rotating frame. (b) Hill's region (schematic, the region in white), which contains a 'neck' about $L_{1}$ and $L_{2}$. (c) The flow in the region near $L_{2}$, showing a periodic orbit around $L_{2}$, a typical asymptotic orbit winding onto the periodic orbit, two transit orbits and two non-transit orbits. A similar figure holds for the region around $L_{1}$.

which is related to the Jacobi constant $C$ by $C=-2 E$. Energy manifolds are 3dimensional surfaces foliating the 4-dimensional phase space. For fixed energy, Poincaré sections are then 2-dimensional, making visualization of intersections between sets in the phase space particularly simple.

Our main concern is the behavior of orbits whose energy is just above that of $L_{2}$. Roughly, we refer to this small energy range as the temporary capture energy range. Fortunately, the temporary capture energy manifolds for the Sun-Earth and Earth-Moon systems intersect in phase space, making a fuel efficient transfer possible.

\subsection{EQUILIBRIUM POINTS}

The PCR3BP has three collinear equilibrium (Lagrange) points which are unstable, but for the cases of interest to mission design, we examine only $L_{1}$ and $L_{2}$ in this paper. Eigenvalues of the linearized equations at $L_{1}$ and $L_{2}$ have one real and one imaginary pair. Roughly speaking, the equilibrium region has the dynamics of a saddle $\times$ harmonic oscillator.

\subsection{HILL'S REGIONS}

The Hill's region which is the projection of the energy manifold onto the position space is the region in the $x y$-plane where the spacecraft is energetically permitted to move around. The forbidden region is the region which is not accessible for the given energy. For an energy value just above that of $L_{2}$, the Hill's region contains a 'neck' about $L_{1}$ and $L_{2}$ and the spacecraft can make transition through these necks. See Figure 3(b). This equilibrium neck region and its relation to the global orbit structure is critical: it was studied in detail by Conley (1968), McGehee (1969), and Llibre et al. (1985). 


\subsection{THE FLOW NEAR $L_{1}$ AND $L_{2}$}

More precisely, in each region around $L_{1}$ and $L_{2}$, there exist four types of orbits (see Figure 3(c)): (1) an unstable periodic Lyapunov orbit (black oval); (2) four cylinders of asymptotic orbits that wind on to or off this period orbit; they form pieces of stable and unstable manifolds; (3) transit orbits which the spacecraft can use to make a transition from one region to the other; for example, passing from the exterior region (outside Earth's orbit) into the Earth temporary capture region (bubble surrounding Earth) via the neck region; (4) non-transit orbits where the spacecraft bounces back to its original region.

\subsection{INVARIANT MANIFOLDS AS SEPARATRICES}

Furthermore, invariant manifold tubes are global objects - they extend far beyond the vicinity of $L_{1}$ and $L_{2}$. These tubes partition the energy manifold and act as separatrices for the flow through the equilibrium region: those inside the tubes are transit orbits and those outside the tubes are non-transit orbits. For example in the Earth-Moon system, for a spacecraft to transit from outside the Moon's orbit to the Moon capture region, it is possible only through the $L_{2}$ periodic orbit stable manifold tube. Hence, stable and unstable manifold tubes control the transport of material to and from the capture region. These tubes can be utilized also for ballistic capture. See Figure 4.

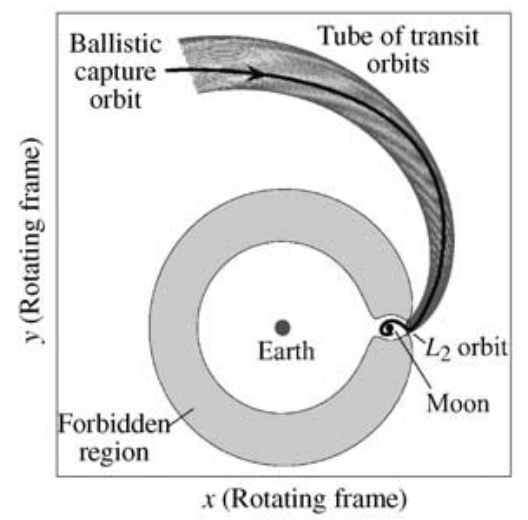

(a)

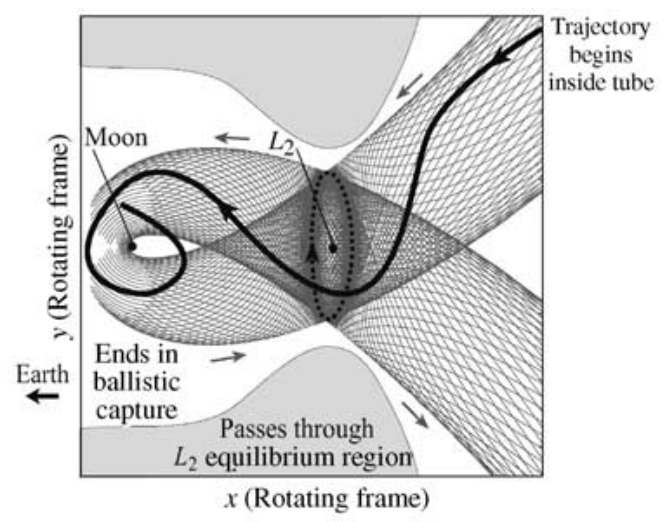

(b)

Figure 4. (a) Trajectories inside the stable manifold tube will transit from outside Moon's orbit to Moon capture region. (b) Trajectory that ends in ballistic capture at the Moon.

\section{Low Energy Transfer to the Moon with Ballistic Capture}

\subsection{TWO COUPLED THREE-BODY MODEL}

By taking full advantage of the dynamics of the 4-body system (Earth, Moon, Sun, and spacecraft), the fuel necessary to transfer from the Earth to the Moon can 
be significantly less than that required by a Hohmann transfer. However, since the structure of the phase space of the 4-body system is poorly understood in comparison with the 3-body system, we initially model it as two coupled planar circular restricted 3-body systems. By doing this, we can utilize the Lagrange point dynamics of both the Earth-Moon-spacecraft and Sun-Earth-spacecraft systems. In this simplified model, the Moon is on a circular orbit about the Earth, the Earth (or rather the Earth-Moon center of mass) is on a circular orbit about the Sun, and the systems are coplanar. In the actual solar system: the Moon's eccentricity is 0.055 , Earth's is 0.017. and the Moon's orbit is inclined to Earth's orbit by $5^{\circ}$. These values are low, so the coupled planar circular 3-body problem is considered a good starting model. An orbit which becomes a real mission is typically obtained first in such an approximate system and then later refined through more precise models which include effects such as out-of-plane motion, eccentricity, the other planets, solar wind, etc. However, tremendous insight is gained by considering a simpler model which reveals the essence of the transfer dynamics.

This is similar to the more standard approach in mission design where the solar system is viewed as a series of 2-body problems where Keplerian theory applies. JPL's spectacular multiple fly-by missions such as Voyager and Galileo are based on this Keplerian decomposition of the solar system. But when one needs to deal with the ballistic capture regime of motion, a 3-body decomposition of the solar system is absolutely necessary.

However, the success of this approach depends greatly on the configuration of the specific four bodies of interest. In order for low energy transfers to take place, the invariant manifold structures of the two 3-body systems must intersect within a reasonable time. Otherwise, the transfer may require an impractically long time of flight. For the Sun-Earth-Moon-spacecraft case, this is not a problem. The overlap of these invariant manifold structures provide the low energy transfers between the Earth and the Moon.

\subsection{CONSTRUCTION OF EARTH-TO-MOON TRANSFER}

The construction is done mainly in the Sun-Earth rotating frame using the Poincaré section $\Gamma$ (along a line of constant $x$-position passing through the Earth). This Poincaré section helps to glue the Sun-Earth Lagrange point portion of the trajectory with the lunar ballistic capture portion. See Figures 7(c) and (d), p. 71.

The basic strategy is to find an initial condition (position and velocity) for a spacecraft on the Poincare section such that when integrating forward, the spacecraft will be guided by the $L_{2}$ Earth-Moon manifold and get ballistically captured by the Moon; when integrating backward, the spacecraft will hug the Sun-Earth manifolds and return to Earth.

We utilize two important properties of the Lagrange point dynamics of the 3body problem. The stable manifold tube is key in targeting a capture orbit for the Earth-Moon portion of the design. The twisting of orbits in the equilibrium region 
is key in finding a fuel efficient transfer for the Sun-Earth Lagrange point portion of the trajectory. For more details, see Koon et al. (2000).

\subsection{LUNAR BALLISTIC CAPTURE PORTION}

Recall that by targeting the region enclosed by the stable manifold tube of the $L_{2}$ Lyapunov orbit in the Earth-Moon system, we can construct an orbit which will get ballistically captured by the Moon. When we transform this Poincare cut of the stable manifold of an Earth-Moon $L_{2}$ Lyapunov orbit into the Poincaré section of the Sun-Earth system, we obtain a closed curve. A point interior to this curve will approach the Moon when integrated forward. See Figure 5. Assuming the Sun is a negligible perturbation to the Earth-Moon-spacecraft 3-body dynamics during this leg of the trajectory, any spacecraft with initial conditions within this closed curve will be ballistically captured by the Moon. 'Ballistic capture by the Moon' means an orbit which under natural dynamics gets within the sphere of influence of the Moon $(20,000 \mathrm{~km})$ and performs at least one revolution around the Moon. In such a state, a slight $\Delta V$ will result in a stable capture (closing off the necks at $L_{1}$ and $L_{2}$ ).

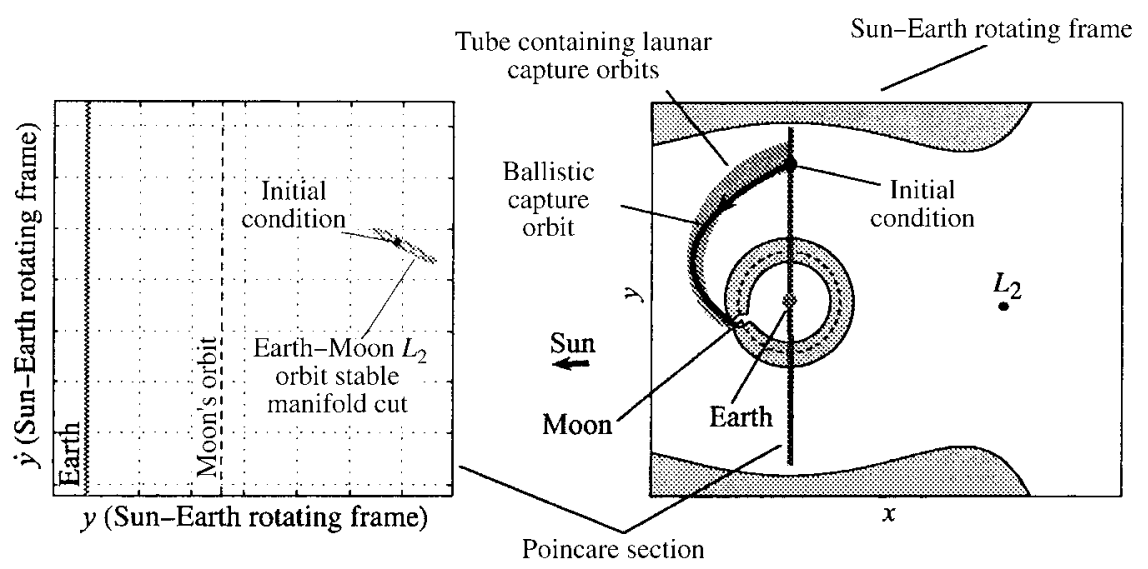

Figure 5. (a) The stable manifold cut of an Earth-Moon $L_{2}$ orbit in the Poincarè section of the Sun-Earth system. (b) A point interior to this cut, with the correct phasing of the Moon, will reach the Moon's ballistic capture region when integrated forward.

\subsection{TWISTING OF ORBITS AND SUN-EARTH LAGRANGE POINT PORTION}

Since the twisting of orbits in the equilibrium region is key in finding the Sun-Earth Lagrange point portion of the design, we would like to review this property briefly. In Koon et al. (2000), we learn that orbits twist in the equilibrium region following roughly the Lyapunov orbit. The amount of twist of an orbit depends sensitively on its distance from the manifold tube. The closer to the manifold tube an orbit begins 
on its approach to the equilibrium region, the more it will be twisted when it exits the equilibrium region. Hence, with small change in the initial condition (such as a small change in velocity at a fixed point), we can change the destination of an orbit dramatically. In fact, we can use this sensitivity to target the spacecraft back to a $200 \mathrm{~km}$ Earth parking orbit.

Look at the Poincaré section $\Gamma$ in Figure 6(a). Notice that how a minute line strip $q_{2} q_{1}$ of orbits just outside of the unstable manifold cut, when integrated backward, gets stretched into a long strip $P^{-1}\left(q_{2}\right) P^{-1}\left(q_{1}\right)$ of orbits that wraps around the whole stable manifold cut. Recall that points on $q_{2} q_{1}$ represent orbits which have the same position but slightly different velocity. But their pre-image $P^{-1}\left(q_{2}\right) P^{-1}\left(q_{1}\right)$ can reach any position on the lower line where the stable manifold tube intersects (see Figure 6(b)).

Pick an energy in the temporary capture range of the Sun-Earth system which has $L_{2}$ orbit manifolds that come near a $200 \mathrm{~km}$ altitude Earth parking orbit. Compute the Poincaré section $\Gamma$ (see Figure 6(a)). The curve on the right is the Poincaré cut of the unstable manifold of the Lyapunov orbit around the Sun-Earth $L_{2}$. Picking an appropriate initial condition just outside this curve, we can backward integrate to produce a trajectory coming back to the Earth parking orbit.
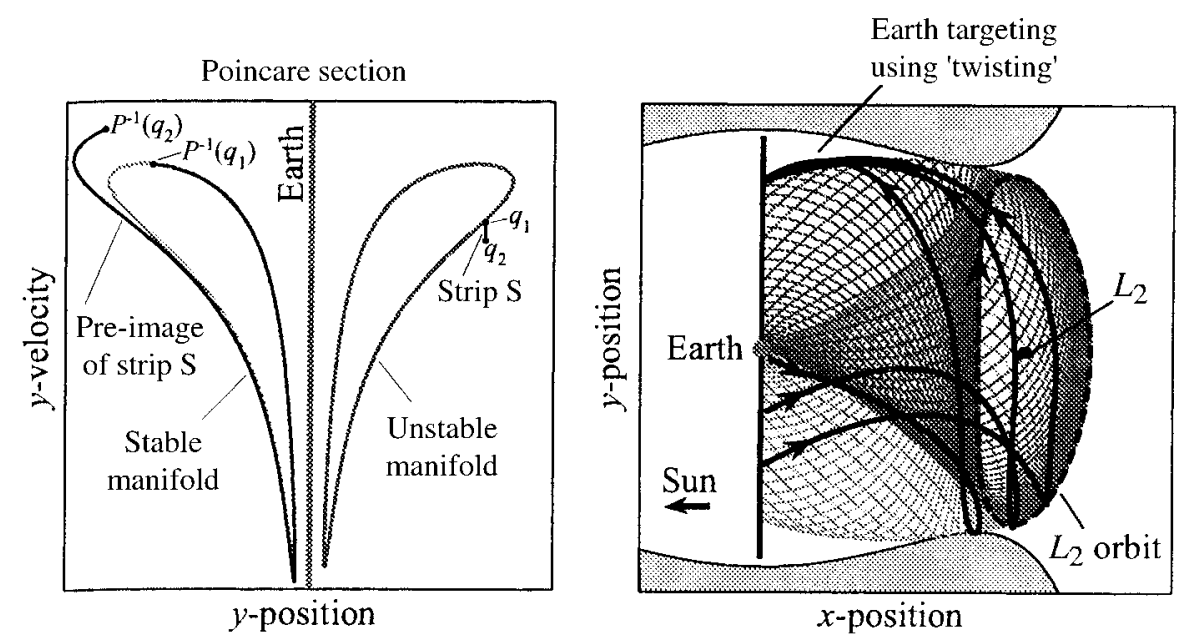

Figure 6. (a) Line strip $q_{2} q_{1}$ outside of unstable manifold cut gets stretched into a long strip $P^{-1}\left(q_{2}\right) P^{-1}\left(q_{1}\right)$ that wraps around stable manifold cut. (b) With infinitesimal changes in velocity, any point near lower tube cross-section can be targeted (integrating backward).

\subsection{CONNECTING THE TWO PORTIONS}

We can vary the phase of the Moon until the Earth-Moon $L_{2}$ manifold cut intersects the Sun-Earth $L_{2}$ manifold cut. See Figures 7(a) and (b). In the region which is in the interior of the gray curve but in the exterior of the black curve, an orbit will get ballistically captured by the Moon when integrated forward; when integrated 


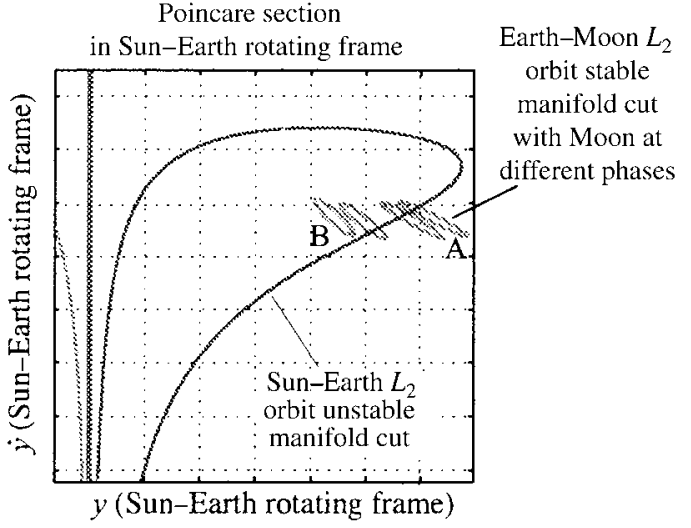

(a)
Stable manifold tube in Earth-Moon rotating frame

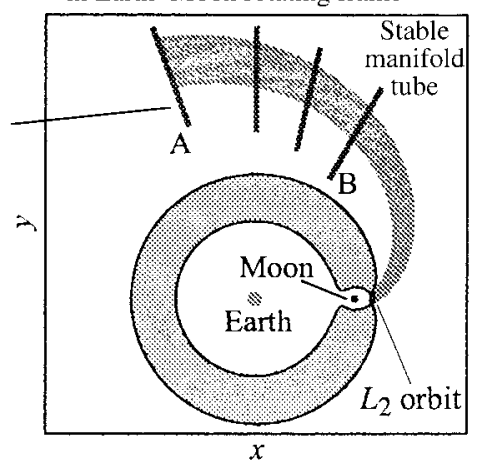

(b)

Sun-Earth $L_{2}$ orbit
unstable manifold cut

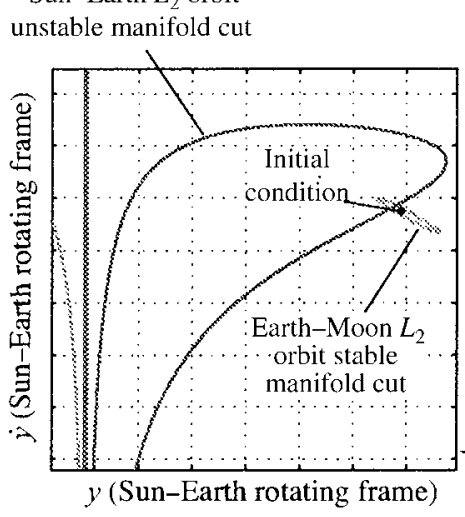

(c)

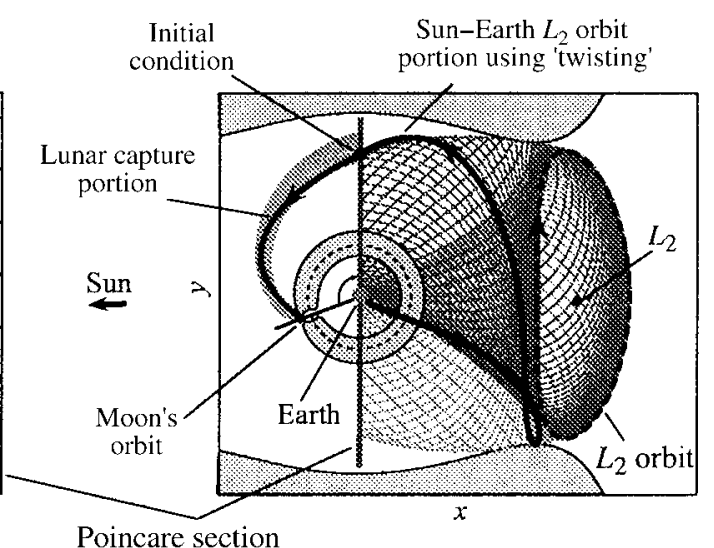

(d)

Figure 7. (a) and (b) Vary the phase of the Moon until Earth-Moon $L_{2}$ manifold cut intersects Sun-Earth $L_{2}$ manifold cut. (c) Pick a point in the interior of gray curve but in the exterior of black curve. (d) An orbit will get ballistically captured by the Moon when integrated foreword; when integrated backward, orbit will hug the invariant manifolds back to the Earth.

backward, the orbit will hug the unstable manifold back to the Sun-Earth $L_{2}$ equilibrium region with a twist, and then hug the stable manifold back towards the position of the Earth parking orbit. See Figures 7(c) and (d).

With only a slight modification (a small mid-course $\Delta V$ of $34 \mathrm{~m} / \mathrm{s}$ at the patch point), this procedure produces a genuine solution integrated in the bi-circular 4body problem. Since the capture at the Moon is natural (zero $\Delta V$ ), the amount of on-board fuel necessary is lowered by about $20 \%$ compared to a traditional Hohmann transfer. This bi-circular solution has been differentially corrected to a fully integrated trajectory with JPL ephemeris using JPL's LTool. This is the subject of a future paper. 


\subsection{WHY DOES IT WORK?}

What follows is a couple of heuristic arguments for using the coupled 3-body model. When outside the Moon's small sphere of influence $(20,000 \mathrm{~km})$, which is most of the pre-capture flight, we can consider the Moon's perturbation on the Sun-Earth-spacecraft 3-body system to be negligible. Thus, we can utilize Sun-Earth Lagrange point invariant manifold structures. The mid-course $\Delta V$ is performed at a point where the spacecraft is re-entering the Earth's sphere of influence $(900,000 \mathrm{~km})$, where we can consider the Sun's perturbation on the EarthMoon-spacecraft 3-body system to be negligible. Thus, Earth-Moon Lagrange point structures can be utilized for the lunar portion of the trajectory.

Moreover, the fact that the patch point $\Delta V$ is so small and may even be eliminated can be understood by considering the following. From a $200 \mathrm{~km}$ circular orbit around the Earth, it requires approximately $3150 \mathrm{~m} / \mathrm{s}$ (provided by the launch vehicle) to reach the Earth-Moon $L_{1}$ and $L_{2}$. For another $50 \mathrm{~m} / \mathrm{s}$, you can reach the Sun-Earth $L_{1}$ and $L_{2}$ ! In other words, a spacecraft needs roughly the same amount of energy to reach the Sun-Earth and the Earth-Moon $L_{1}$ and $L_{2}$. This fortuitous coincidence is what enables these low energy lunar transfer and capture orbits.

\section{Conclusion}

We have laid bare the dynamical mechanism for a Hiten-like mission. The theory of Lagrange point dynamics of the 3-body system developed in Koon et al. (2000) is crucial in understanding this problem. In many previous applications of dynamical systems theory to mission design, the focus has been on using the trajectory arcs on the invariant manifolds as initial guesses for the desired end-to-end trajectory. In this paper, we have shown that the tubular regions enclosed by the manifolds, the regions exterior to the manifolds, as well as the manifolds themselves all may be used to advantage depending on the desired characteristics of the final trajectory. Mission designers with this knowledge can pick and choose to their hearts' content, an infinite variety of trajectories to suit almost any purpose at hand.

\section{References}

Belbruno, E. A. and Miller, J. K.: 1993, 'Sun-perturbed Earth-to-Moon transfers with ballistic capture', J. Guid. Cont. Dyn. 16, 770-775.

Conley, C.: 1968, 'Low energy transit orbits in the restricted three-body problem', SIAM J. Appl. Math. 16, 732-746.

Koon W. S., Lo, M. W., Marsden, J. E. and Ross, S. D.: 2000, 'Heteroclinic connections between Lyapunov orbits and resonance transitions in celestial mechanics', Chaos 10, 427-469.

Llibre, J., Martinez, R. and Simó, C.: 1985, 'Transversality of the invariant manifolds associated to the Lyapunov family of periodic orbits near $L 2$ in the restricted three-body problem', J. Differ. Eq. 58, 104-156. 
McGehee, R. P.: 1969, 'Some Homoclinic Orbits for the Restricted Three Body Problem', PhD Thesis, University of Wisconsin, Madison, Wisconsin.

Szebehely, V.: 1967, Theory of Orbits, The Restricted Problem of Three Bodies, Academic Press, New York and London. 